\title{
Simplest equation method to look for exact solutions of nonlinear differential equations
}

\author{
N.A. Kudryashov \\ Department of Applied Mathematics \\ Moscow Engineering and Physics Institute \\ (State university) \\ 31 Kashirskoe Shosse, 115409 \\ Moscow, Russian Federation
}

\begin{abstract}
New method is presented to look for exact solutions of nonlinear differential equations. Two basic ideas are at the heart of our approach. One of them is to use the general solutions of the simplest nonlinear differential equations. Another idea is to take into consideration all possible singularities of equation studied. Application of our approach to search exact solutions of nonlinear differential equations is discussed in detail. The method is used to look for exact solutions of the Kuramoto - Sivashinsky equation and the equation for description of nonlinear waves in a convective fluid. New exact solitary and periodic waves of these equations are given.
\end{abstract}

Keywords: exact solution, nonlinear differential equation, travelling wave, simplest equation method.

PACS: $02.30 . \mathrm{Hq}$ - Ordinary differential equations

\section{Introduction}

One of the most exciting recent advances of nonlinear science and theoretical physics has been a development of methods to look for exact solutions for nonlinear differential equations. This is important because many mathematical models are described by nonlinear differential equations.

The inverse scattering transform [1] and the direct method by R. Hirota [2] are known as impressive methods to search solutions of exactly solvable differential equations.

The singular manifold method [3-5], the transformation method [6-8], the tanh-function method [9-12], the sh-function method [13,14] and the Weierstrass function method $[15,16]$ are useful in many applications to search exact solutions of nonlinear partially solvable differential equations.

In this paper we present new approach to look for exact solutions of nonlinear differential equations. We take two basic ideas for our method into account. 
The first idea is to apply the simplest nonlinear differential equations (the Riccati equation, the equation for the Jacobi elliptic fuction, the equation for the Weierstrass ellipic function and so on) that have lesser order then the equation studied. The second idea is to use all possible singularities of the equation studied.

There are two advantages of our method. The first advantage is that our approach generalizes a number of methods that were applied before. The second advantage of the method is simplicity of realization.

We apply our approach to look for exact solutions of the Kuramoto - Sivashinsky equation [17-19] and for the equation at the description of nonlinear waves on the surface in a convective fluid $[20,21]$.

The outline of this paper is as follows. New approach to look for exact solution of nonlinear differential equation is discussed in section 2. Application of our approach to look for exact solitary and periodic waves of the Kuramoto Sivashinsky equation are given in sections 3 and 4 . Exact solitary and periodic waves of the equation for nonlinear waves in a convective fluid are presented in sections 5 and 6 .

\section{Method applied}

Consider the nonlinear ordinary differential equation in the polynomial form

$M[y]=0$

Let us assume we look for exact solutions of equation (2.1). Take the nonlinear ordinary differential equation of lesser order than equation (2.1)

$E_{n}[Y]=0$

Any nonlinear ordinary differential equation (2.2) of lesser order than (2.1) with known general solution we call as the simplest equation.

The first example of the simplest equation is the Riccati equation

$E_{1}[Y]=Y_{z}+Y^{2}-a Y-b=0$

The second example of the simplest equation is the equation for the Jacobi elliptic function

$E_{2}[Q]=Q_{z}^{2}-Q^{4}-a Q^{3}-b Q^{2}-c Q-d=0$

The third example of the simplest equation is the equation for the Weierstrass elliptic function

$E_{3}[R]=R_{z}^{2}-4 R^{3}-a R^{2}-b R-c=0$

We emphasize that the general solutions of equations (2.3) and (2.4) have the first order singularities but the general solution of equation (2.5) has the second order singularity. We are going to use these properties of equations (2.3), (2.4) and (2.5). 
Let us show that the general solution of equation (2.4) is written via the Jacobi elliptic function $\operatorname{sn}(m z, k)$. Equation (2.4) can be presented in the form

$Q_{z}^{2}=\left(Q-Q_{1}\right)\left(Q-Q_{2}\right)\left(Q-Q_{3}\right)\left(Q-Q_{4}\right)$

where $Q_{1}, Q_{2}, Q_{3}$ and $Q_{4}$ are roots of the equation

$Q^{4}+a Q^{3}+b Q^{2}+c Q+d=0$

Taking new variable

$Q^{2}=\frac{\left(Q_{2}-Q_{4}\right)\left(W(z)-Q_{1}\right)}{\left(Q_{1}-Q_{4}\right)\left(W(z)-Q_{2}\right)}$

with

$k^{2}=\frac{\left(Q_{2}-Q_{3}\right)\left(Q_{1}-Q_{4}\right)}{\left(Q_{1}-Q_{3}\right)\left(Q_{2}-Q_{4}\right)}$

$m^{2}=\frac{1}{4}\left(Q_{2}-Q_{4}\right)\left(Q_{1}-Q_{3}\right)$

into consideration we have the equation

$\left(\frac{d W}{d z}\right)^{2}=\left(1-W^{2}\right)\left(1-k^{2} W^{2}\right)$

with the general solution

$W(z)=\operatorname{sn}(m z, k)$

The general solution of equation (2.5) is expressed via the Weierstrass elliptic function because having used new variable

$R=\wp-\frac{a^{2}}{12}$

we obtain the equation for the Weierstrass elliptic function [22]

$\wp^{2}=4 \wp^{3}-g_{2} \wp-g_{3}$,

where $g_{2}=\frac{a^{2}}{12}-b, \quad g_{3}=\frac{a b}{12}-c-\frac{a^{3}}{216}$.

Suppose we can find the relation between solutions of equations (2.1) and (2.2)

$y=F(Y)$ 
In addition, suppose that substitution (2.15) into equation (2.1) leads to the relation in the form

$M[F(Y)]=\hat{A} E_{n}[Y]$

where $\hat{A}$ is an operator that should be found.

we can see from relation (2.16) that for any solution of the simplest equation (2.2) there exists a special solution of equation (2.1) by the formula (2.15).

Later we will show that there is the transformation (2.15) that allows us to obtain relation (2.16).

Consider our method in detail. It contains the following steps.

The first step. Determination of the dominant terms and the singularity order for the solution of equation (2.1).

To find the dominant terms of equation (2.1) we substitute

$y=A_{k}\left(z-z_{0}\right)^{k}$

into all terms of equation (2.1).

It should be noted that the first step of our method correspond to the first step of the Painleve test that is used to analyze equation (2.1) on the Painleve property [23]. We have to compare the degrees of all terms of equation (2.1) and take two or more of them with the least degree. Let $N$ be the least degree of the dominant terms of equation (2.1) and denote by $n$ the value of $k$ that corresponds to the least degree.

The value of $k$ that leads to the terms of the least degree by substitution of (2.17) into equation (2.1) we call as the singularity order of the solution of equation (2.1).

Our method can be applied when $n$ is integer. In the case of non - integer value of $n$ one can try to transfer equation (2.1).

The second step. Arrangement of formula (2.15) as polynomial dependence on the general solution of the simplest equation (2.2). This formula can be written taking into consideration the singularity orders for the solutions of equations (2.1) and (2.2).

Taking equation (2.3) into consideration in this paper we firstly introduce and use new formula (2.15) to look for exact solutions of nonlinear differential equations that takes the form

$y(z)=A_{0}+A_{1} Y+\ldots+A_{n} Y^{n}+B_{1}\left(\frac{Y_{z}}{Y}\right)+\ldots+B_{n}\left(\frac{Y_{z}}{Y}\right)^{n}$

where $Y(z)$ is the general solution of equation (2.3), coefficients $A_{k}(k=0, \ldots, n)$ and $B_{k}(k=1, \ldots, n)$ are unknown coefficients that should be found.

It should be noted that assuming $B_{k}=0(k=1, \ldots, n)$ in formula (2.18) and $a=0$ in equation (2.3) our approach is reduced to the tanh-function method to look for exact solution of nonlinear differential equation (2.1). For $a=0$ in equation (2.3) we obtain the method of the hyperbolic functions from formula (2.18) to look for exact solutions. 
Taking equation (2.4) into consideration we also firstly introduce and we apply formula (2.15) in the form

$$
\begin{aligned}
y(z)= & A_{0}+A_{1} Q+\ldots+A_{n} Q^{n}+\left(B_{1}+\ldots+B_{n-1} Q^{n-2}\right) Q_{z}+ \\
& +D_{1}\left(\frac{Q_{z}}{Q}\right)+D_{2}\left(\frac{Q_{z}}{Q}\right)^{2}+\ldots+D_{n}\left(\frac{Q_{z}}{Q}\right)^{n},
\end{aligned}
$$

where $Q(z)$ is the general solution of equation (2.4), $A_{k}(k=0, \ldots, n), B_{k}(k=$ $1, \ldots, n-1)$ and $D_{k}(k=1, \ldots, n)$ are coefficients that should be found. For $B_{k}=0,(k=0, \ldots, n)$ and $D_{k}=0(k=1, \ldots, n)$ formula (2.19) can be reduced to the sn-function method to look for exact solutions of equation (2.1).

Taking equation (2.5) into account we can also suggest formula (2.15) to look for exact solutions of nonlinear differential equations in the form

$$
\begin{aligned}
& y(z)=A_{0}+D_{1}\left(\frac{R_{z}}{R}\right)+D_{2}\left(\frac{R_{z}}{R}\right)^{2}+\ldots+D_{n}\left(\frac{R_{z}}{R}\right)^{n}+ \\
& +A_{1} R+B_{1} R_{z}+\left(A_{2} R+B_{2} R_{z}\right) R+\left(A_{3} R+B_{3} R_{z}\right) R^{2}+\ldots,
\end{aligned}
$$

where $R(z)$ is the general solution of equation (2.5). The last term in formula (2.20) is determined by the singularity order of the solution of equation (2.1). If $n=2 m$ then the last term is $A_{m} R^{m}$. In the case $n=2 m-1$ the last term in formula (2.20) is $B_{m} R^{m-1} R_{z}$.

Assuming $A_{k}=0(k=2, \ldots, n), D_{k}=0(k=1, \ldots, n), B_{k}=0(k=$ $2, \ldots, n)$ from formula (2.20) we have the approach by N.A. Kudryashov [15] that was used to find exact solutions of nonlinear differential equation. Using $A_{k}=0(k=2, \ldots, n), D_{k}=0(k=2, \ldots, n)$ and $B_{k}=0(k=1, \ldots, n)$ from expression (2.20) we obtain the method by A.V. Porubov [16].

The third step. Determination of coefficients $A_{k}(k=0, \ldots, n), B_{k}(k=$ $1, \ldots, n)$ and $D_{k}(k=1, \ldots, n)$ in formulas (2.18), (2.19) and (2.20).

Using formulas (2.18), (2.19) or (2.20) for finding exact solutions of equation (2.1) we chose the type of solution for equation (2.1). If we use formula (2.18) we can find exact solutions of equation (2.1) in the form of solitary waves. Using formula (2.19) we can look for special periodic solutions expressed via the Jacobi elliptic functions. If we use formula (2.20) we can obtain periodic exact solutions expressed via the Weierstrass elliptic function.

Coefficients $A_{k}(k=0, \ldots, n), B_{k}(k=1, \ldots, n)$ and $D_{k}(k=1, \ldots, n)$ are determined after substitution of formulas (2.18), (2.19) and (2.20) into equation (2.1). As a result of this substitution we obtain the equation with derivatives of $Y$ (or $Q$ and $R$ ) with respect to $z$. To find coefficients $A_{k}(k=0, \ldots, n)$, $B_{k}(k=1, \ldots, n)$ and $D_{k}(k=1, \ldots, n)$ we take the following simple theorems into consideration.

Theorem 2.1. Let $Y(z)$ be a solution of equation (2.3). Than the equations

$Y_{z z}=2 Y^{3}-3 a Y^{2}+\left(a^{2}-2 b\right) Y+a b$ 


$$
\begin{aligned}
& Y_{z z z}=-6 Y^{4}+12 Y^{3} a+\left(8 b-7 a^{2}\right) Y^{2}+ \\
& \left(-8 a b+a^{3}\right) Y-2 b^{2}+a^{2} b \\
& Y_{z z z z}=24 Y^{5}-60 Y^{4} a+\left(50 a^{2}-40 b\right) Y^{3}+ \\
& +\left(60 a b-15 a^{3}\right) Y^{2}+\left(16 b^{2}-22 a^{2} b+a^{4}\right) Y-8 a b^{2}+a^{3} b \\
& Y_{z z z z z}=-120 Y^{6}+360 Y^{5} a+\left(-390 a^{2}+240 b\right) Y^{4}+ \\
& +\left(180 a^{3}-480 a b\right) Y^{3}+\left(-136 b^{2}-31 a^{4}+292 a^{2} b\right) Y^{2}+ \\
& +\left(136 a b^{2}-52 a^{3} b+a^{5}\right) Y-22 a^{2} b^{2}+a^{4} b+16 b^{3}
\end{aligned}
$$

have special solutions that are expressed via the general solution of equation (2.3).

Proof. Theorem 2.1 is proved by differentiation of (2.3) with respect to $z$ and substitution $Y_{z}$ from equation (2.3) into expressions obtained.

Theorem 2.2. Let $Q(z)$ be a solution of equation (2.4). Than the equations

$$
\begin{aligned}
& Q_{z z}=2 Q^{3}+\frac{3}{2} a Q^{2}+b Q+\frac{1}{2} c \\
& Q_{z z z}=6 Q^{2} Q_{z}+3 a Q(z) Q_{z}+b Q_{z} \\
& Q_{z z z z}=24 Q^{5}+30 a Q^{4}+\left(\frac{15}{2} a^{2}+20 b\right) Q^{3}+ \\
& +(152 a b+15 c) Q^{2}+\left(b^{2}+\frac{9}{2} a c+12 d\right) Q+3 a d+\frac{1}{2} b c \\
& Q_{z z z z z}=120 Q^{4} Q_{z}+120 a Q^{3} Q_{z}+3\left(\frac{15}{2} a^{2}+20 b\right) Q^{2} Q_{z}+ \\
& +2\left(\frac{15}{2} a b+15 c\right) Q(z) Q_{z}+\left(b^{2}+\frac{9}{2} a c+12 d\right) Q_{z}
\end{aligned}
$$

have special solutions that are expressed via the general solution of equation (2.4).

Proof. Theorem 2.2 is proved along similar lines than theorem 2.1.

Theorem 2.3. Let $R(z)$ be a solution of equation (2.5). Than the equations

$$
R_{z z}=6 R^{2}+a R+\frac{1}{2} b
$$


$R_{z z z}=12 R R_{z}+a R_{z}$

$R_{z z z z}=120 R^{3}+30 a R^{2}+\left(a^{2}+18 b\right) R+\frac{1}{2} a b+12 c$

$R_{z z z z z}=360 R^{2} R_{z}+60 a R R_{z}+\left(a^{2}+18 b\right) R_{z}$

have special solutions that are expressed via the general solution of equation (2.5).

Proof. Theorem 2.3 is proved in a manner like theorem 2.1.

Substituting dependence $y$ on $Y$ (2.18) into equation (2.1) and taking theorem 2.1 into account we obtain

$M[y]=\sum_{k=0}^{2 N} P_{k}\left(a, b, A_{0}, \ldots, A_{n}, B_{1}, \ldots, B_{n}\right) Y^{k-N}$

Consider the system of algebraic equations with respect to $a, b, A_{k}(k=$ $0, \ldots, n)$ and $B_{k}(k=1, \ldots, n)$

$P_{k}\left(a, b, A_{0}, \ldots, A_{n}, B_{1}, \ldots, B_{n}\right)=0(k=0, \ldots, 2 N)$

As a consequence of the choice for the dominant terms of equation (2.1) we have $A_{n} \neq 0$ at the first step of the method. Assume that there are solutions of the system of equations (2.34). If equations (2.34) are satisfied then

$M[y]=0$

and $y$ by formula (2.18) is the solution of equation (2.1). Here $Y(z)$ is the general solution of equation (2.3).

Substituting (2.19) into equation (2.1) and taking theorem 2.2 into consideration we have the equality in the form

$$
\begin{gathered}
M[y]=\sum_{k=-N}^{N} P_{k}\left(a, b, c, d, A_{0}, \ldots, A_{n}, B_{1}, \ldots, B_{n-1}, D_{1}, \ldots, D_{n},\right) Q^{k}+ \\
+\sum_{k=2-N}^{N-2} S_{k}\left(a, b, c, d, A_{0}, \ldots, A_{n}, B_{1}, \ldots, B_{n-1}, D_{1}, \ldots, D_{n}\right) Q^{k} Q_{z} .
\end{gathered}
$$

Suppose we have solutions of the systems of equation

$$
\begin{gathered}
P_{k}\left(a, b, c, d, A_{0}, \ldots, A_{n}, B_{1}, \ldots, B_{n-1}, D_{1}, \ldots, D_{n}\right)=0, \\
(k=0, \ldots, 2 N)
\end{gathered}
$$




$$
\begin{gathered}
S_{k}\left(a, b, c, d, A_{0}, \ldots, A_{n}, B_{1}, \ldots, B_{n-1}, D_{1}, \ldots, D_{n}\right)=0, \\
(k=1, \ldots, 2 N-4)
\end{gathered}
$$

then $y$ expressed by the formula (2.19) allows us to have exact solutions of equation (2.1).

Substituting (2.20) into equation (2.1) and using theorem 2.3 we obtain the relation in the form

$$
\begin{gathered}
M[y]=\sum_{k=-L}^{L} P_{k}\left(a, b, c, D_{1}, \ldots, D_{n}, A_{0}, A_{1} \ldots B_{1}, B_{2} \ldots\right) R^{k}+ \\
\quad+\sum_{k=-M}^{M} S_{k}\left(a, b, c, D_{1}, \ldots, D_{n}, A_{0}, A_{1} \ldots B_{1}, B_{2} \ldots\right) R^{k} R_{z}
\end{gathered}
$$

If $N=2 m$ then we take in (2.39) $L=N$ and $M=N-2$. In the case $N=2 m-1$ we need to take $M=N$ and $L=N-1$. If we have solutions for coefficients $D_{1}, \ldots, D_{n} ; A_{0}, A_{2}, \ldots$ and $B_{1}, B_{2}, \ldots$ of the system of the equations

$P_{k}\left(a, b, c, D_{1}, \ldots, D_{n}, A_{0}, A_{1}, \ldots, B_{1}, B_{2}, \ldots\right)=0, k=0, \ldots, 2 L$

$S_{k}\left(a, b, c, D_{1}, \ldots, D_{n}, A_{0}, A_{1}, \ldots, B_{1}, B_{2}, \ldots\right)=0, k=0, \ldots, 2 M$

we obtain exact solution of equation in the form (2.20) that is expressed via the Weierstrass solution.

In this manner the method applied allows us to look for exact solutions of equation (2.1). Let us consider the application of our method to look for exact solitary and periodic waves of nonlinear differential equations.

\section{Exact solitary waves of the Kuramoto - Sivashin- sky equation}

Let us apply our approach to look for exact solitary waves of the Kuramoto Sivashinsky equation. This equation has the form

$u_{t}+u u_{x}+\alpha u_{x x}+\beta u_{x x x}+\gamma u_{x x x x}=0$

Nonlinear evolution equation (3.1) describes nonlinear waves in active - dissipative dispersive media. For $\alpha=0, \gamma=0$ and $\beta \neq 0$ from equation (3.1) we have the famous Korteveg - de Vries equation. The Causchy problem for this equation can be solved by the inverse scattering transform [1]. In the case $\beta=0$, $\gamma=0$ and $\alpha \neq 0$ from equation (3.1) we get the Burgers equation that can be 
linearized by the Cole - Hopf transformation into the heat equation $[24,25]$. At $\alpha \neq 0, \beta \neq 0$ and $\gamma \neq 0$ equation (3.1) is not integrable because this one does not pass the Painleve test [23]. However there are several special solutions of this equation.

Exact solitary waves of equation at $\alpha \neq 0, \beta \neq 0$ and $\gamma \neq 0$ was firstly found in [4] at $\beta=0 ; \beta= \pm 4 \sqrt{\alpha \gamma} ; \beta= \pm 12 \sqrt{\alpha \gamma / 47} ; \beta= \pm 16 \sqrt{\alpha \gamma / 73}$. Later these solutions were rediscovered more than once. Let us show that using our approach we can obtain more general solitary waves.

Using new variables

$u=u^{\prime} \alpha \sqrt{\frac{\alpha}{\gamma}} \quad x=x^{\prime} \sqrt{\frac{\gamma}{\alpha}}, \quad t=t^{\prime} \frac{\gamma^{2}}{\alpha}, \quad \sigma=\frac{\beta}{\sqrt{\alpha \gamma}}$,

(the primes of the variables are omitted) we have equation in the form

$u_{t}+u u_{x}+u_{x x}+\sigma u_{x x x}+u_{x x x x}=0$

Taking the travelling wave into consideration

$u(x, t)=y(z), \quad z=x-C_{0} t$

we obtain from equation (3.3)

$C_{1}-C_{0} y+y_{z}+\sigma y_{z z}+y_{z z z}+\frac{1}{2} y^{2}=0$

The equation of the dominant terms is

$y_{z z z}+\frac{1}{2} y^{2}=0$

Substituting (2.17) into equation (3.5) we can see that the general solution of equation (3.5) has the singularity order equals three and we take $n=3$ in formula (2.18). Therefore we will look for exact solution of equation (3.5) in the form

$y(z)=A_{0}+A_{1} Y+A_{2} Y^{2}+A_{3} Y^{3}+\frac{B_{1} Y_{z}}{Y}+\frac{B_{2} Y_{z}^{2}}{Y^{2}}+\frac{B_{3} Y_{z}^{3}}{Y^{3}}$

It should be noted that formula (3.7) can be written in the form

$$
\begin{gathered}
y(z)=A_{0}-2 B_{2} b+\left(A_{3}-B_{3}\right) Y^{3}+\left(B_{2}+A_{2}\right) Y^{2}+ \\
+\left(A_{1}-B_{1}+3 B_{3} b\right) Y+\frac{B_{1} b-3 B_{3} b^{2}}{Y}+\frac{B_{2} b^{2}}{Y^{2}}+\frac{B_{3} b^{3}}{Y^{3}}
\end{gathered}
$$


Substituting (3.8) into equation (3.5) we have

$B_{3}^{(1)}=120, \quad B_{3}^{(2)}=0$

Consider the case: $B_{3}=B_{3}^{(1)}=120$. We obtain

$B_{2}=-15 \sigma, \quad B_{1}=-\frac{15}{76} \sigma^{2}+\frac{60}{19}+240 b$

$A_{0}=C_{0}+\frac{7 \sigma}{76}-\frac{13}{608} \sigma^{3}-20 b \sigma$

$A_{3}^{(1)}=240, \quad A_{3}^{(2)}=120$

Assuming $A_{3}=A_{3}^{(1)}=240$ we have additionally

$A_{2}=0, \quad A_{1}=-\frac{15}{38} \sigma^{2}+\frac{120}{19}-240 b$

We also obtain

$b_{1,2}=-\frac{5}{4864} \sigma^{2}+\frac{5}{304} \pm \frac{1}{4864} \sqrt{549 \sigma^{4}-3584 \sigma^{2}+9216}$

Taking equation (3.14) into account we find two variants values $\sigma$. The first variant has

$\sigma_{1}^{(1)}=0, \quad \sigma_{2,3}^{(1)}= \pm 4$

and the second variant takes the form

$\sigma_{1}^{(2)}=0, \quad \sigma_{2,3}^{(2)}= \pm 4, \quad \sigma_{4,5}^{(2)}= \pm \frac{12}{\sqrt{47}}, \quad \sigma_{6,7}^{(2)}= \pm \frac{16}{\sqrt{73}}$

These values $\sigma$ corresponds to two variants values $b$. The first variant is

$b_{1}^{(1)}=\frac{11}{304}, \quad b_{2,3}^{(1)}=\frac{1}{16}$

and the second variant takes the form

$b_{1}^{(2)}=-\frac{1}{304}, \quad b_{2,3}^{(2)}=\frac{1}{1168}, \quad b_{4,5}^{(2)}=\frac{1}{752}, \quad b_{6,7}^{(2)}=-\frac{1}{16}$ 
As a result of calculations for these values $\sigma$ and $b$ we have the following special solutions of equation (3.5) for the first variant

$$
\begin{gathered}
y_{1}^{(1)}(z)=C_{0}-\frac{45 \sqrt{11}}{152 \sqrt{19}}\left(U+U^{-1}\right)+\frac{165 \sqrt{11}}{152 \sqrt{19}}\left(U^{3}+U^{-3}\right), \\
U=\tanh \left(\frac{\sqrt{11}}{4 \sqrt{19}}\left(z-C_{2}\right)\right) \\
y_{2,3}^{(1)}(z)=C_{0} \pm \frac{3}{2}-\frac{15}{8}\left(U+U^{-1}\right) \mp \frac{15}{4}\left(U^{2}+U^{-2}\right)+ \\
+\frac{15}{8}\left(U^{3}+U^{-3}\right), \quad U=\tanh \left(\frac{1}{4}\left(z-C_{2}\right)\right)
\end{gathered}
$$

Special solutions for the second variant take the form

$$
\begin{gathered}
y_{1}^{(2)}(z)=C_{0}-\frac{135}{152 \sqrt{19}}\left(U-U^{-1}\right)-\frac{15}{152 \sqrt{19}}\left(U^{3}-U^{-3}\right) \\
U=\tan \left(\frac{1}{4 \sqrt{19}}\left(z-C_{2}\right)\right) \\
y_{2,3}^{(2)}(z)=C_{0} \pm \frac{30}{\sqrt{73}}+\frac{345}{584 \sqrt{73}}\left(U+U^{-1}\right) \mp \frac{15}{\sqrt{73}}\left(U^{2}+U^{-2}\right)+ \\
+\frac{15}{584 \sqrt{73}}\left(U^{3}+U^{-3}\right), \quad U=\tanh \left(\frac{1}{4 \sqrt{73}}\left(z-C_{2}\right)\right) \\
y_{4,5}^{(2)}(z)=C_{0}+\frac{225}{376 \sqrt{47}}\left(U+U^{-1}\right) \mp \frac{45}{188 \sqrt{47}}\left(U^{2}+U^{-2}\right) \pm \\
\pm \frac{45}{94 \sqrt{47}}+\frac{15}{376 \sqrt{47}}\left(U^{3}+U^{-3}\right), \quad U=\tanh \left(\frac{1}{4 \sqrt{47}}\left(z-C_{2}\right)\right) \\
y_{6,7}^{(2)}(z)=C_{0} \mp \frac{7}{2}-\frac{15}{8}\left(U-U^{-1}\right) \mp \frac{15}{4}\left(U^{2}+U^{-2}\right) \\
-\frac{15}{8}\left(U^{3}-U^{-3}\right), \quad \tan \left(\frac{1}{4}\left(z-C_{2}\right)\right)
\end{gathered}
$$

Constant $C_{1}$ in the case of the first variant is determined by the formulas

$$
C_{1}^{(1)}=\frac{1}{2} C_{0}^{2}-\frac{4950}{6859}, \quad C_{2,3}^{(1)}=\frac{1}{2} C_{0}^{2}-18
$$


Constant $C_{1}$ for the second variant is expressed by the formulas

$$
\begin{gathered}
C_{1}^{(2)}=\frac{1}{2} C_{0}^{2}+\frac{450}{6859}, \quad C_{2,3}^{(2)}=\frac{1}{2} C_{0}^{2}-\frac{4050}{389017} \\
C_{4,5}^{(2)}=\frac{1}{2} C_{0}^{2}-\frac{1800}{103823}, \quad C_{6,7}^{(2)}=\frac{1}{2} C_{0}^{2}-8
\end{gathered}
$$

Assuming $A_{3}=A_{3}^{(2)}=120$ we have

$$
\begin{aligned}
& A_{2}=15 \sigma, \quad A_{1}=-\frac{15}{76} \sigma^{2}+\frac{60}{19}-120 b \\
& b_{1}=\frac{7}{380}-\frac{13}{3040} \sigma^{2}, \quad b_{2}=0
\end{aligned}
$$

We also obtain six values for $\sigma$

$$
\sigma_{1,2}= \pm \frac{16}{\sqrt{73}}, \quad \sigma_{3,4}= \pm \frac{12}{\sqrt{47}}, \quad \sigma_{5,6}= \pm \frac{2 \sqrt{14}}{\sqrt{13}}
$$

Constant $C_{1}$ takes values

$C_{1,2}=\frac{1}{2} C_{0}^{2}-\frac{4050}{389017}, \quad C_{3,4}=\frac{1}{2} C_{0}^{2}-\frac{1800}{103823}, \quad C_{5,6}=\frac{1}{2} C_{0}^{2}$

Special solutions of the Kuramoto - Sivashinsky equation can be written in the form

$$
\begin{gathered}
y_{1,2}(z)=C_{0}+\frac{15}{\sqrt{73}}\left(5 U^{-1} \mp 4 U^{-2}+U^{-3} \pm 4\right), \\
U=\tanh \left(\frac{1}{2 \sqrt{73}}\left(z-C_{2}\right)\right) \\
y_{3,4}(z)=C_{0}+\frac{15}{\sqrt{47}}\left(3 U^{-1} \mp 3 U^{-2}+U^{-3} \pm 3\right), \\
U=\tanh \left(\frac{1}{2 \sqrt{47}}\left(z-C_{2}\right)\right)
\end{gathered}
$$

For $\sigma=\sigma_{5,6}$ we have trivial solutions that are constants.

In the case $B_{3}=0$ we obtain

$$
B_{2}=0, \quad B_{1}=0, \quad A_{3}^{(1)}=120, \quad A_{3}^{(2)}=0
$$


Assuming $A_{3}=A_{3}^{(1)}=120$ we have

$$
\begin{aligned}
& A_{2}=-15 \sigma, \quad A_{1}=\frac{60}{19}-\frac{15}{76} \sigma^{2}-120 b \sigma \\
& A_{0}=C_{0}+\frac{7}{76} \sigma+10 \sigma b-\frac{13}{608} \sigma^{3} \\
& b_{1,2}=\frac{5}{76}-\frac{5}{1216} \sigma^{2} \pm \frac{1}{1216} \sqrt{9216-3584 \sigma^{2}+549 \sigma^{4}}
\end{aligned}
$$

There are two variants values of $\sigma$ corresponding to $b_{1,2}$ :

$$
\sigma_{1}^{(1)}=0, \quad \sigma_{2,3}^{(1)}= \pm 4
$$

and

$$
\sigma_{1}^{(2)}=0, \quad \sigma_{2,3}^{(2)}= \pm \frac{16}{\sqrt{73}},, \quad \sigma_{4,5}^{(2)}= \pm \frac{12}{\sqrt{47}},, \quad \sigma_{6,7}^{(2)}= \pm 4
$$

Special solutions of the Kuramoto - Sivashinsky equation in the case of the first variant take the form

$$
\begin{aligned}
& y_{1}^{(1)}(z)=C_{0}+\frac{15 \sqrt{11}}{\sqrt{19}}\left(9 U-11 U^{3}\right), \quad U=\tanh \left(\frac{\sqrt{11}}{2 \sqrt{19}}\left(z-C_{2}\right)\right) \\
& y_{2,3}^{(1)}(z)=C_{0} \pm 9+15\left(U \mp U^{2}-U^{3}\right), \quad U=\tanh \left(\frac{1}{2}\left(z-C_{2}\right)\right)
\end{aligned}
$$

Constant $C_{1}$ in equation (3.5) for the special solutions $y_{i}^{(1)}(i=1, \ldots, 3)$ is given by formula 3.25.

Special solutions of the second variant are determined by the formulas

$$
\begin{gathered}
y_{1}^{(2)}(z)=C_{0}+\frac{15}{\sqrt{19}}\left(3 U+U^{3}\right), \quad U=\tan \left(\frac{1}{2 \sqrt{19}}\left(z-C_{2}\right)\right) \\
y_{2,3}^{(2)}(z)=C_{0}-\frac{15}{\sqrt{73}}\left(5 U \pm 4 U^{2}+U^{3} \mp 4\right), \\
U=\tanh \left(\frac{1}{2 \sqrt{73}}\left(z-C_{2}\right)\right)
\end{gathered}
$$




$$
\begin{gathered}
y_{4,5}^{(2)}(z)=C_{0}-\frac{15}{\sqrt{47}}\left(3 U \pm 3 U^{2}+U^{3} \mp 3\right), \\
U=\tanh \left(\frac{1}{2 \sqrt{47}}\left(z-C_{2}\right)\right) \\
y_{6,7}^{(2)}(z)=C_{0} \mp 11+15\left(U \mp U^{2}+U^{3}\right), \quad U=\tan \left(\frac{1}{2}\left(z-C_{2}\right)\right)
\end{gathered}
$$

Constant $C_{1}$ in equation (3.5) for the special solutions $y_{j}^{(2)}(j=1, \ldots, 7)$ is given by formula (3.26).

The special solutions (3.39) - (3.44) of the Kuramoto - Sivashinsky equation were found in $[4,10]$. The special solutions of the Kuramoto - Sivashinsky equation (3.1) in the form of the solitary waves (3.19), (3.20), (3.21), (3.22), (3.23), (3.24), (3.31) and (3.32) are new.

\section{Exact periodic waves of the Kuramoto - Sivashin- sky equation}

Periodic exact solutions of the Kuramoto - Sivashinsky equation (3.1) were firstly obtained at $\beta= \pm 4 \sqrt{\alpha \gamma}$ taking the Weierstrass elliptic function into account in [15]. Let us show that our method allows to look for exact solutions that are expressed via the Jacobi elliptic functions.

Consider the equation

$\gamma y_{z z z}+\beta y_{z z}+\alpha y_{z}+\frac{1}{2} y^{2}-C_{0} y+C_{1}=0$.

This equation can be found from equation (3.1) if we use travelling wave (3.4).

Let us look for exact solutions of equation 4.1) in the form

$$
\begin{gathered}
y(z)=A_{0}+A_{1} Q+A_{2} Q^{2}+A_{3} Q^{3}+B_{1} Q_{z}+B_{2} Q Q_{z}+ \\
+D_{1} \frac{Q_{z}}{Q}+D_{2} \frac{Q_{z}^{2}}{Q^{2}}+D_{3} \frac{Q_{z}^{3}}{Q^{3}}
\end{gathered}
$$

where $Q(z)$ is solution of equation (2.4). There is the case $D_{3}=0, D_{2}=0, D_{1}=$ 0 of special solution and we consider this variant of formula (4.2). Substituting (4.2) into equation (4.1) and taking the theorem 2.2 into consideration we have

$$
\begin{aligned}
& B_{2}=-60 \gamma, \quad A_{3}= \pm 60 \gamma \\
& B_{1}= \pm \frac{15}{2} \beta-15 \gamma a, \quad A_{2}= \pm 45 \gamma a-\frac{15}{2} \beta
\end{aligned}
$$




$$
\begin{aligned}
& A_{1}=-\frac{15}{4} \beta a \pm 30 \gamma b \pm \frac{10}{3} \alpha \mp \frac{5}{24} \frac{\beta^{2}}{\gamma} \\
& A_{0}=C_{0}-\frac{5}{4} \beta b+\frac{1}{6} \frac{\alpha \beta}{\gamma} \pm \frac{5}{6} \alpha a \pm 15 \gamma c \mp \frac{5}{96} \frac{\beta^{2} a}{\gamma}-\frac{5}{192} \frac{\beta^{3}}{\gamma^{2}} \\
& d=\frac{1}{4} a c-\frac{1}{12} b^{2}+\frac{1}{12} \frac{\alpha^{2}}{\gamma^{2}} \\
& C_{1}=15 b \alpha^{2}+\frac{1}{2} C_{0}{ }^{2}-13 \frac{\alpha^{3}}{\gamma}-20 \gamma^{2} b^{3}+\frac{135}{2} \gamma^{2} b c a-\frac{135}{2} \gamma^{2} c^{2}+ \\
& +\frac{45}{8} \gamma^{2} a^{2} b^{2}-\frac{45}{8} a^{2} \alpha^{2}-\frac{135}{8} \gamma^{2} a^{3} c
\end{aligned}
$$

There is the additional condition for solution of equation (4.1): $\beta= \pm 4 \sqrt{\alpha \gamma}$.

Exact solutions of equation (4.1) at $\beta=\sqrt{\alpha \gamma}$ in the form of the periodic wave take the form

$$
\begin{gathered}
y(z)=C_{0} \pm 15 \gamma c-5 b \sqrt{\alpha \gamma}-\frac{\alpha^{3 / 2}}{\sqrt{\gamma}}-15 a \sqrt{\alpha \gamma} Q \pm \\
\pm 30 b \gamma Q-30 \sqrt{\alpha \gamma} Q^{2} \pm 45 a \gamma Q^{2} \pm 60 \gamma Q^{3}-15 a \gamma Q_{z} \pm \\
\pm 30 \sqrt{\alpha \gamma} Q_{z}-60 \gamma Q Q_{z}
\end{gathered}
$$

Here $Q(z)$ is the general solution of the equation for the Jacobi elliptic function in the form

$$
Q_{z}^{2}-Q^{4}-a Q^{3}-b Q^{2}-c Q-\frac{1}{12} \gamma^{2} \alpha^{2}+\frac{1}{12} b^{2}-\frac{1}{4} a c=0
$$

There is exact solutions of equation (4.1) at $\alpha=\beta=0$ in the form

$$
\begin{gathered}
y(z)=C_{0} \pm 15 \gamma c \pm 30 b \gamma Q \pm 45 a \gamma Q^{2} \pm 60 \gamma Q^{3}- \\
-15 a \gamma Q_{z}-60 \gamma Q Q_{z}
\end{gathered}
$$

Special solutions in the form of periodic waves (4.9) and (4.11) of the $\mathrm{Ku}$ ramoto - Sivashinsky equation are expressed via the Jacobi elliptic function are new. 


\section{Exact solitary waves of the equation in a con- vective fluid}

The equation

$\gamma y_{z z z}+\beta y_{z z}+\alpha y_{z}+\frac{1}{2} \varepsilon y^{2}+\chi y y_{z}-C_{0} y+C_{1}=0$

can be obtained from the nonlinear evolution equation

$u_{t}+\varepsilon u u_{x}+\chi\left(u u_{x}\right)_{x}+\alpha u_{x x}+\beta u_{x x x}+\gamma u_{x x x x}=0$

that occurs at the description of nonlinear waves in a convecting fluid [20]. Assuming $\chi=0$ in (5.1) we have the Kuramoto - Sivashinsky equation and below we suppose $\chi=1$.

Equation (5.1) does not pass the Painleve test and this one is not exactly solvable equation but it has several special solutions that were found in $[9,16]$.

Let us consider equation (5.1) using our method to look for new exact solitary waves. Equation for the dominant terms has the form

$\gamma y_{z z z}+y y_{z}=0$

The singularity order for solution of equation (5.1) is equal to $n=2$ and we can look for exact solution in the form

$y(z)=A_{0}+A_{1} Y+A_{2} Y^{2}+B_{1} \frac{Y_{z}}{Y}+B_{2} \frac{Y_{z}^{2}}{Y^{2}}$,

where $Y(z)$ is the general solution of the Riccati equation (2.3). Substituting expression (5.4) into equation (5.1) we have the following values of coefficients:

$B_{2}^{(1)}=-12 \gamma, \quad B_{2}^{(2)}=0$.

Considering the first case $B_{2}=B_{2}^{(1)}=-12 \gamma$ we obtain

$A_{2}^{(1)}=12 \gamma, \quad A_{2}^{(2)}=0$

Take $A_{2}=A_{2}^{(1)}=12 \gamma$. In this case we have

$$
\begin{aligned}
& B_{1}=\frac{12}{5} \beta+24 \gamma a-\frac{12}{5} \varepsilon \gamma \\
& A_{1}=-24 \gamma a+\frac{12}{5} \beta-\frac{12}{5} \varepsilon \gamma
\end{aligned}
$$




$$
\begin{aligned}
& A_{0}=\frac{23}{25} \beta \varepsilon+\frac{12}{5} \gamma a \varepsilon-\frac{24}{25} \gamma \varepsilon^{2}-4 \gamma a^{2}+\frac{1}{25} \frac{\beta^{2}}{\gamma}- \\
& -\frac{12}{5} \beta a-\alpha-16 \gamma b \\
& C_{0}=\frac{1}{125} \frac{\beta^{3}}{\gamma^{2}}-\frac{126}{125} \gamma \varepsilon^{3}+\frac{4}{5} \gamma \varepsilon a^{2}-\frac{4}{5} \beta a^{2}-\frac{3}{125} \frac{\varepsilon \beta^{2}}{\gamma}+\frac{128}{125} \varepsilon^{2} \beta- \\
& -\alpha \varepsilon+\frac{4}{5} \gamma \varepsilon b-\frac{4}{5} \beta b \\
& b^{(1)}=\frac{1}{100} \frac{\beta^{2}}{\gamma^{2}}-\frac{1}{50} \frac{\beta \varepsilon}{\gamma}+\frac{1}{100} \varepsilon^{2}-a^{2}, \quad b^{(2)}=0 \\
& C_{1}=\frac{409}{1250} \beta^{2} \varepsilon^{3}-\frac{18}{625} \frac{\beta^{4} \varepsilon}{\gamma^{2}}+\frac{72}{625} \frac{\beta^{3} \varepsilon^{2}}{\gamma}-\frac{553}{625} \gamma \beta \varepsilon^{4}+ \\
& +\frac{1}{2} \varepsilon \alpha^{2}+\gamma \alpha \varepsilon^{3}-\alpha \varepsilon^{2} \beta+\frac{589}{1250} \gamma^{2} \varepsilon^{5} .
\end{aligned}
$$

Solution of equation (5.1) in this case has the form

$$
\begin{gathered}
y(z)=\frac{12}{5} \beta a-12 \gamma a^{2}-\frac{22}{25} \gamma \varepsilon^{2}-\alpha-\frac{12}{5} \gamma a \varepsilon+ \\
+\frac{3}{25} \frac{\beta^{2}}{\gamma}+\frac{19}{25} \beta \varepsilon+\frac{3}{125} \frac{(\beta-\varepsilon \gamma+10 \gamma a)(\beta-\varepsilon \gamma-10 \gamma a)^{2}}{\gamma^{2} Y}- \\
-\frac{3}{2500} \frac{(\beta-\varepsilon \gamma-10 \gamma a)^{2}(\beta-\varepsilon \gamma+10 \gamma a)^{2}}{\gamma^{3} Y^{2}}
\end{gathered}
$$

where $Y(z)$ takes the form

$$
Y(z)=a+\frac{1}{10}\left(\frac{\beta}{\gamma}-\epsilon\right) \tanh \left(\frac{1}{10}\left(\frac{\beta}{\gamma}-\epsilon\right)\left(z-C_{2}\right)\right)
$$

We found new exact solutions (5.13) in the form of the solitary wave with 
two arbitrary constants $a$ and $C_{2}$ that satisfies equation (5.1) in the form

$$
\begin{gathered}
\gamma y_{z z z}+\beta y_{z z}+\alpha y_{z}+y y_{z}+\frac{1}{2} \varepsilon y^{2}-\frac{4}{5}\left(\gamma \varepsilon b-\beta a^{2}-\beta b\right) y- \\
-\left(\gamma \varepsilon a^{2}+\frac{3}{125} \frac{\varepsilon \beta^{2}}{\gamma}+\frac{128}{125} \varepsilon^{2} \beta-\alpha \varepsilon-\frac{126}{125} \gamma \varepsilon^{3}+\frac{1}{125} \frac{\beta^{3}}{\gamma^{2}}\right) y+ \\
+\frac{589}{1250} \gamma^{2} \epsilon^{5}-\frac{18}{625} \frac{\beta^{4} \varepsilon}{\gamma^{2}}+\frac{72}{625} \frac{\beta^{3} \varepsilon^{2}}{\gamma}+\frac{409}{1250} \beta^{2} \varepsilon^{3}-\frac{553}{625} \gamma \beta \varepsilon^{4}+ \\
+\frac{1}{2} \varepsilon \alpha^{2}+\gamma \varepsilon^{3} \alpha-\varepsilon^{2} \beta \alpha=0
\end{gathered}
$$

Assuming $\alpha=1, \beta=1, \gamma=1$ and $\varepsilon=2$ we have equation (5.15) in simple form

$y_{z z z}+y_{z z}+y_{z}+y y_{z}+y^{2}-\frac{16}{5} b y+\frac{751}{125} y+\frac{10589}{625}=0$

In the case $B_{2}=B_{2}^{(1)}=-12 \gamma$ and $A_{2}^{(2)}=0$ we have $a=0$ and

$$
\begin{aligned}
& B_{1}=\frac{12}{5}(\beta-\varepsilon \gamma), \quad A_{1}=\frac{24}{5}(\beta-\varepsilon \gamma) \\
& A_{0}=-\frac{24}{25} \gamma \varepsilon^{2}-16 \gamma b+\frac{1}{25} \frac{\beta^{2}}{\gamma}-\alpha+\frac{23}{25} \varepsilon \beta \\
& C_{0}=\frac{16}{5} \gamma \varepsilon b-\frac{16}{5} \beta b-\frac{126}{125} \gamma \varepsilon^{3}-\frac{3}{125} \frac{\beta^{2} \varepsilon}{\gamma}+\frac{1}{125} \frac{\beta^{3}}{\gamma^{2}}- \\
& \quad-\alpha \varepsilon+\frac{128}{125} \varepsilon^{2} \beta \\
& C_{1}=\frac{72}{625} \frac{\varepsilon^{2} \beta^{3}}{\gamma}+\frac{589}{1250} \gamma^{2} \varepsilon^{5}-\frac{553}{625} \gamma \varepsilon^{4} \beta+\frac{409}{1250} \varepsilon^{3} \beta^{2}-\frac{18}{625} \frac{\beta^{4} \varepsilon}{\gamma^{2}}+ \\
& +\gamma \alpha \varepsilon^{3}+\frac{1}{2} \varepsilon \alpha^{2}-\alpha \varepsilon^{2} \beta
\end{aligned}
$$

We have the exact solitary wave in the form

$$
\begin{gathered}
y(z)=\frac{3}{50} \frac{\beta^{2}}{\gamma}-\alpha-\frac{47}{50} \gamma \varepsilon^{2}+\frac{22}{25} \varepsilon \beta-12 \gamma Y^{2} \\
+\frac{3}{500} \frac{(\beta-\varepsilon \gamma)^{3}}{\gamma^{2} Y}-\frac{3}{40000} \frac{(\beta-\varepsilon \gamma)^{4}}{g^{3} Y^{2}}+\frac{12}{5}(\beta-\varepsilon \gamma) Y
\end{gathered}
$$


where $Y(z)$ satisfies the equation

$$
Y_{z}+Y^{2}-\frac{1}{400} \varepsilon^{2}+\frac{1}{200} \frac{\varepsilon \beta}{\gamma}-\frac{1}{400} \frac{\beta^{2}}{\gamma^{2}}=0
$$

and takes the form

$$
Y(z)=\frac{1}{20}\left(\frac{\beta}{\gamma}-\varepsilon\right) \tanh \left(\frac{1}{20}\left(\frac{\beta}{\gamma}-\epsilon\right)\left(z-C_{2}\right)\right) .
$$

Exact solution (5.1) is the special solution of the following equation

$$
\begin{gathered}
\gamma y_{z z z}+\beta y_{z z}+\alpha y_{z}+y y_{z}+\frac{1}{2} \varepsilon y^{2}-\alpha \epsilon y+\frac{1}{2} \varepsilon \alpha^{2}+\alpha \varepsilon^{2} \beta+\gamma \alpha \varepsilon^{3}- \\
-\left(\frac{16}{5} \gamma \varepsilon b-\frac{16}{5} \beta b-\frac{126}{125} \gamma \varepsilon^{3}-\frac{3}{125} \frac{\beta^{2} \varepsilon}{\gamma}+\frac{1}{125} \frac{\beta^{3}}{\gamma^{2}}+\frac{128}{125} \varepsilon^{2} \beta\right) y- \\
-\frac{553}{625} \gamma \varepsilon^{4} \beta+\frac{409}{1250} \varepsilon^{3} \beta^{2}-\frac{18}{625} \frac{\beta^{4} \varepsilon}{\gamma^{2}}+\frac{72}{625} \frac{\varepsilon^{2} \beta^{3}}{\gamma}+\frac{589}{1250} \gamma^{2} \varepsilon^{5}=0
\end{gathered}
$$

Assuming $\alpha=1, \beta=1, \gamma=1$ and $\varepsilon=2$ from (5.24) we have the equation in the form

$$
y_{z z z}+y_{z z}+y_{z}+y y_{z}+y^{2}-\frac{16}{5} b y+\frac{751}{125} y+\frac{10589}{625}=0
$$

Consider the case $B_{2}=B_{2}^{(1)}=0$ and $A_{2}=A_{2}^{(1)}=-12 \gamma$. We obtain

$$
\begin{aligned}
& B_{1}=0, \quad A_{1}=24 \gamma a+\frac{12}{5}(\beta-\varepsilon \gamma) \\
& A_{0}=8 \gamma b-\frac{12}{5} \beta a+\frac{1}{25} \frac{\beta^{2}}{\gamma}+\frac{23}{25} \beta \varepsilon-\alpha-4 \gamma a^{2}+\frac{12}{5} \gamma a \varepsilon-\frac{24}{25} \gamma \varepsilon^{2} \\
& C_{0}=\frac{4}{5} \gamma \varepsilon a^{2}+\frac{4}{5} \gamma \varepsilon b-\frac{126}{125} \gamma \varepsilon^{3}-\frac{4}{5} \beta a^{2}-\frac{4}{5} \beta b-\frac{3}{125} \frac{\varepsilon \beta^{2}}{\gamma}+ \\
& \quad+\frac{128}{125} \varepsilon^{2} \beta-\varepsilon \alpha+\frac{1}{125} \frac{\beta^{3}}{\gamma^{2}} \\
& b=\frac{1}{100} \varepsilon^{2}-a^{2}-\frac{1}{50} \frac{\beta \varepsilon}{\gamma}+\frac{1}{100} \frac{\beta^{2}}{\gamma^{2}}
\end{aligned}
$$




$$
\begin{gathered}
C_{1}=\frac{1}{2} \varepsilon \alpha^{2}+\gamma \varepsilon^{3} \alpha-\varepsilon^{2} \beta \alpha+\frac{409}{1250} \varepsilon^{3} \beta^{2}-\frac{18}{625} \frac{\varepsilon \beta^{4}}{\gamma^{2}}- \\
-\frac{553}{625} \gamma \varepsilon^{4} \beta+\frac{72}{625} \frac{\varepsilon^{2} \beta^{3}}{\gamma}+\frac{589}{1250} \gamma^{2} \varepsilon^{5}
\end{gathered}
$$

As a result of calculations we have the exact solitary waves in the form that were found in $[9,16]$

$$
\begin{gathered}
y(z)=-12 \gamma Y^{2}+\left(24 \gamma a+\frac{12}{5} \beta-\frac{12}{5} \varepsilon \gamma\right) Y- \\
-\frac{12}{5} \beta a+\frac{3}{25} \frac{\beta^{2}}{\gamma}+\frac{19}{25} \beta \varepsilon-\alpha-12 \gamma a^{2}+\frac{12}{5} \gamma a \varepsilon-\frac{22}{25} \gamma \varepsilon^{2}
\end{gathered}
$$

where $Y(z)$ satisfies the equation

$$
Y_{z}+Y^{2}-2 a Y-\frac{1}{100} \varepsilon^{2}+a^{2}+\frac{1}{50} \frac{\beta \varepsilon}{\gamma}-\frac{1}{100} \frac{\beta^{2}}{\gamma^{2}}=0
$$

and is expressed by the function

$$
Y(z)=a+\frac{1}{10}\left(\frac{\beta}{\gamma}-\varepsilon\right) \tanh \left(\frac{1}{10}\left(\frac{\beta}{\gamma}-\varepsilon\right)\left(z-C_{2}\right)\right)
$$

Equation (5.1) can be presented in the form

$$
\begin{gathered}
\gamma y_{z z z}+\beta y_{z z}+\alpha y_{z}+y y_{z}+\frac{1}{2} \varepsilon y^{2}+\gamma \varepsilon^{3} \alpha-\varepsilon^{2} \beta \alpha+ \\
+\left(\frac{126}{125} \gamma \varepsilon^{3}+\frac{4}{5} \beta a^{2}+\frac{4}{5} \beta b+\frac{3}{125} \frac{\varepsilon \beta^{2}}{\gamma}-\frac{128}{125} \varepsilon^{2} \beta\right) y- \\
-\left(\varepsilon \alpha+\frac{4}{5} \gamma \varepsilon a^{2}+\frac{4}{5} \gamma \varepsilon b+\frac{1}{125} \frac{\beta^{3}}{\gamma^{2}}\right) y+\frac{1}{2} \varepsilon \alpha^{2}+ \\
+\frac{409}{1250} \varepsilon^{3} \beta^{2}-\frac{18}{625} \frac{\varepsilon \beta^{4}}{\gamma^{2}}-\frac{553}{625} \gamma \varepsilon^{4} \beta+\frac{589}{1250} \gamma^{2} \varepsilon^{5}-\frac{72}{625} \frac{\varepsilon^{2} \beta^{3}}{\gamma}=0
\end{gathered}
$$

Assuming $\alpha=1, \beta=1, \gamma=1$ and $\varepsilon=2$ from (5.34) we have the equation in simple form

$$
y_{z z z}+y_{z z}+y_{z}+y y_{z}+y^{2}-\frac{4}{5} a^{2} y-\frac{4}{5} b y+\frac{751}{125} y+\frac{5589}{625}=0
$$

These exact solitary waves can be used to analyze nonlinear wave processes at the description in a convective fluid. 


\section{Exact periodic waves of the equation in a con- vective fluid}

Consider the equation for waves in a convective fluid again and find the exact solutions in the form of periodic waves. We use the following formula

$$
y(z)=A_{0}+A_{1} Q+A_{2} Q^{2}+D_{1} Q_{z}+B_{1} \frac{Q_{z}}{Q}+B_{2} \frac{Q_{z}^{2}}{Q^{2}}
$$

where $Q(z)$ is the general solution of equation (2.4).

Substituting (6.1) into (5.1) we have $B_{2}^{(1)}=-12 \gamma, B_{2}^{(2)}=0$.

First of all let us consider the first case $B_{2}=B_{2}^{(1)}=-12 \gamma$.

We have

$d=0, \quad c=0, \quad A_{2}=6 \gamma$

$D_{1}= \pm 6 \gamma, \quad B_{1}=\frac{6}{5}(\varepsilon \gamma-\beta), \quad A_{1}=9 a \gamma \mp \frac{6}{5}(\varepsilon \gamma-\beta)$

$A_{0}=11 b \gamma-\alpha+\frac{23}{25} \beta \varepsilon+\frac{\beta^{2}}{25 \gamma}-\frac{24}{25} \gamma \varepsilon^{2}$

$C_{0}=\frac{\varepsilon \beta^{2}}{25 \gamma}-\varepsilon \alpha-b \varepsilon \gamma-\frac{24}{25} \gamma \varepsilon^{3}+\frac{23}{25} \beta \varepsilon^{2}, \quad b=\frac{(b-\varepsilon g)^{2}}{25 \gamma^{2}}$

$C_{1}=\frac{409}{1250} \varepsilon^{3} \beta^{2}-\varepsilon^{2} \beta \alpha-\frac{18}{625} \frac{\varepsilon \beta^{4}}{\gamma^{2}}+\frac{72}{625} \frac{\varepsilon^{2} \beta^{3}}{\gamma}+\varepsilon^{3} \gamma \alpha+$

$$
+\frac{1}{2} \varepsilon \alpha^{2}+\frac{589}{1250} \varepsilon^{5} \gamma^{2}-\frac{553}{625} \varepsilon^{4} \gamma \beta
$$

The exact periodic waves are determined by the formula

$$
\begin{aligned}
y(z)= & \beta \varepsilon-\alpha-\gamma \varepsilon^{2}+\left( \pm \frac{6}{5} \beta \mp \frac{6}{5} \varepsilon \gamma-3 \gamma a\right) Q- \\
& -6 \gamma Q^{2} \pm 6 \gamma Q_{z}+\frac{6}{5}(\varepsilon \gamma-\beta) \frac{Q_{z}}{Q},
\end{aligned}
$$


where $Q(z)$ is the general solution of equation (2.4). Equation (5.1) in this case can be written in the form

$$
\begin{gathered}
\gamma y_{z z z}+\beta y_{z z}+\alpha y_{z}+\frac{1}{2} \varepsilon y^{2}+y y_{z}+\varepsilon \alpha y+\gamma \varepsilon^{3} y-\beta \varepsilon^{2} y- \\
-\varepsilon^{2} \beta \alpha+\varepsilon^{3} \gamma \alpha+\frac{1}{2} \varepsilon \alpha^{2}+\frac{72}{625} \frac{\varepsilon^{2} \beta^{3}}{\gamma}-\frac{553}{625} \varepsilon^{4} \gamma \beta+ \\
+\frac{409}{1250} \varepsilon^{3} \beta^{2}+\frac{589}{1250} \varepsilon^{5} \gamma^{2}-\frac{18}{625} \frac{\varepsilon \beta^{4}}{\gamma^{2}}=0 .
\end{gathered}
$$

Assuming $\alpha=1, \beta=1, \gamma=1$ and $\varepsilon=1$ we have simple form of equation (6.8)

$y_{z z z}+y_{z z}+y_{z}+\frac{1}{2} y^{2}+y y_{z}+y+\frac{1}{2}=0$.

Consider the second case $B_{2}=B_{2}^{(2)}=0$. We have

$d=0, \quad B_{1}^{(1)}=\beta, \quad B_{1}^{(2)}=0$

Assuming $B_{1}=B_{1}^{(1)}=\beta$ we obtain

$c=0, \quad A_{2}=-6, \gamma \quad D_{1}= \pm 6 \gamma$

$A_{1}= \pm \frac{8}{3} \beta \mp 2 \varepsilon \gamma-3 a \gamma, \quad \beta=\frac{6}{11} \varepsilon \gamma$

$A_{0}=-b \gamma-\frac{54}{121} \varepsilon^{2} \gamma-\alpha$

$C_{0}=-\frac{54}{121} \varepsilon^{3} \gamma-\varepsilon \alpha-b \varepsilon \gamma, \quad b=\frac{1}{121} \varepsilon^{2}$

$C_{1}=\frac{2989}{29282} \varepsilon^{5} \gamma^{2}+\frac{5}{11} \varepsilon^{3} \gamma \alpha+\frac{1}{2} \varepsilon \alpha^{2}$

In this case we have special solution in the form

$$
\begin{gathered}
y(z)=-\frac{5}{11} \varepsilon^{2} \gamma-\alpha-\left( \pm \frac{6}{11} \varepsilon \gamma+3 a \gamma\right) Q-6 \gamma Q^{2}+ \\
\pm 6 \gamma Q_{z}+\frac{6}{11} \varepsilon \gamma \frac{Q_{z}}{Q} .
\end{gathered}
$$


Equation (5.15) for this case takes the form

$$
\begin{gathered}
\gamma y_{z z z}+\frac{6}{11} \varepsilon \gamma y_{z z}+\alpha y_{z}+\frac{1}{2} \varepsilon y^{2}+y y_{z}+\frac{5}{11} \varepsilon^{3} \gamma y+\varepsilon \alpha y+ \\
+\frac{5}{11} \varepsilon^{3} \gamma \alpha+\frac{1}{2} \varepsilon \alpha^{2}+\frac{2989}{29282} \varepsilon^{5} \gamma^{2}=0 .
\end{gathered}
$$

In the case $\alpha=1, \beta=1, \gamma=1$ and $\varepsilon=1$ we have simple equation

$y_{z z z}+y_{z z}+y_{z}+\frac{11}{12} y^{2}+y y_{z}+\frac{1001}{216} y+\frac{90695}{15552}=0$.

Assuming $B_{2}=0$ and $B_{1}=0$ we have

$$
\begin{aligned}
& A_{2}=-6 \gamma, \quad D_{1}= \pm 6 \gamma, \quad A_{1}=\mp 2 \varepsilon \gamma \pm 2 \beta-3 a \gamma \\
& \beta=\varepsilon \gamma \quad A_{0}=-\alpha-b \gamma, \quad C_{0}=-\varepsilon \alpha \\
& C_{1}=\frac{3}{2} \varepsilon \gamma^{2} a c-\frac{1}{2} b^{2} \varepsilon \gamma^{2}+\frac{1}{2} \varepsilon \alpha^{2}-6 \varepsilon \gamma^{2} d
\end{aligned}
$$

Special solution in this case takes the form

$$
y(z)=-\gamma b-\alpha-3 a \gamma Q-6 \gamma Q^{2} \pm 6 \gamma Q_{z}
$$

where $Q(z)$ satisfies equation

$$
Q_{z}^{2}-Q^{4}-a Q^{3}-b Q^{2}-c Q-d=0
$$

Equation (5.15) in this case can be written in the form

$$
\begin{gathered}
\gamma y_{z z z}+\varepsilon \gamma y_{z z}+\alpha y_{z}+\frac{1}{2} \varepsilon y^{2}+y y_{z}+\varepsilon \alpha y+\frac{3}{2} \varepsilon \gamma^{2} a c+ \\
+\frac{1}{2} \varepsilon \alpha^{2}-\frac{1}{2} \varepsilon \gamma^{2} b^{2}-6 \varepsilon \gamma^{2} d=0 .
\end{gathered}
$$

For $\alpha=1, \beta=1, \gamma=1$ and $\varepsilon=1$ we have simple equation

$$
y_{z z z}+y_{z z}+y_{z}+\frac{1}{2} y^{2}+y y_{z}+y+\frac{1}{2}-\frac{1}{2} b^{2}-6 d+\frac{3}{2} a c=0
$$

Special solutions (6.7), (6.16) and (6.22) of equation (5.1) are new. 


\section{Conclusion}

Let us emphasize in brief the results of this work. We have presented new approach to look for exact solutions of nonlinear differential equations. Two basic ideas were taken into account for our method. The first idea was to use the simplest nonlinear ordinary differential equation with known general solution for the finding new special solutions. The second idea was to take all possible singularities of solution of equation studied into consideration. Using these ideas we have suggested new formulas to search exact solutions of nonlinear differential equations in the form of solitary and periodic waves.

Our approach was applied to look for exact solutions of the Kuramoto Sivashinsky equation and the equation for description nonlinear waves in a convective fluid. These equations are noninegrable equations but they are very popular at the description of nonlinear waves in the nonlinear science. New exact solutions in the form of solitary and periodic waves of these nonlinear differential equations were found.

\section{Acknowledgments}

This work was supported by the International Science and Technology Center under Project No 1379-2.

\section{References}

[1] C.S. Gardner, J.M. Greene, M.D. Kruskal and R.R. Miura, Phys. Rev. Lett., 19 (1967) 1095 - 1097

[2] R. Hirota, Phys. Rev. Lett., 27 (1971) 1192 - 1194

[3] J. Weiss, M. Tabor, G. Carnevalle, J. Math. Phys. 24, 1983, 522-526

[4] N.A. Kudryashov, 1988, Journal of Applied Mathematics and Mekhanics, 52, 361-365

[5] R. Conte and M. Musette, J. Phys. A.: Math. Gen. 22, 1989, 169-177

[6] S.R. Choudhury, 1997, Phys. Lett. A., 159, 311-317

[7] N.A. Kudryashov, 1991, Phys Lett. A., 155, 269-275

[8] M. Musette and R. Conte, Physica D, 181, 2003,70-79

[9] S.Y. Lou, G. Huang, H. Ruan, J. Phys. A.: Math. Gen., 24, 1991, 587-590

[10] N.A.Kudryashov, and E.D. Zargaryan, J. Phys. A. Math. and Gen. 29, $1996,8067-8077$

[11] E.G. Fan, Phys Lett. A., 227, 2000, 212-218

[12] S.A. Elwakil, S.K. Ellabany, M.A. Zahran et al., 2002, Phys. Lett. A., 299, $179-188$

[13] S.K. Liu, Z.T. Fu, S.D. Liu, Q. Zhao, Phys. Lett. A., 289, 2001, 69-74 
[14] Z. Y. Yan, Chaos Soliton Fractals 15(3), 2003, 575-583

[15] N.A. Kudryashov, Phys Lett. A., 147, 1990, 287-291

[16] A.V. Porubov, J. Phys. A.: Math. Gen., 26, 1993, L707-L800

[17] P.S. Landa, Nonlinear Oscillations and waves in dynamical syctems, 1996, Kluver Academic Publishers, 538 p.

[18] Y. Kuramoto and T. Tsuzuki, 1976 Prog. Theor. Phys, 55, 356

[19] G.I. Sivashinsky , 1982, 4, 227-235

[20] H. Aspe and M.C. Depassier, 1990, Phys. Rev. A., 41, 3125

[21] A. Garazo and M.G. Velarde, 1991, Phys. Fluids A, 3, 2295

[22] N.I. Akhiezer, Elements of theory of elliptic functions, Moscow, OGIZ (1948) 292 p (in Russian)

[23] R. Conte, 1999 The Painleve property, one century later, CRM series in mathematical physics, Springer - Verlag, New York, 1999, 77-180

[24] E. Hopf, Commun. Pure and Appl. Math., 3, 1950, 201-230

[25] J.D. Cole, Quart. Appl. Math., 9, 1951, 225-236 